\title{
Highly Reduced Saturation Magnetization in Epitaxially Grown Ferrimagnetic Heusler Thin Films
}

Chungman Kim ${ }^{\dagger}$, Woosuk Yoo ${ }^{\dagger}$, Hyun-Woo Bang ${ }^{\dagger}$, Sunghun Lee ${ }^{\dagger, \dagger}$, Yunchang Park ${ }^{\S}$, Young Haeng Lee ${ }^{\|}$, Joonyoung Choi ${ }^{\square}$, Younjung Jo ${ }^{\square}$, Kyujoon Lee ${ }^{\&}$, and Myung-Hwa Jung,,*

†Department of Physics, Sogang University, Seoul 04107, Republic of Korea

Department of Physics and Astronomy, Sejong University, Seoul 05006, Republic of Korea

$\S$ National Nanofab Center, Daejeon 34141, Republic of Korea

"Korea Institute of Science and Technology, Seoul 02792, Republic of Korea

$\square$ Department of Physics, Kyungpook National University, Daegu 41566, Republic of Korea

\&Institute of Physics, Johannes Gutenberg University Mainz, Mainz 55128, Germany

*E-mail: mhjung@sogang.ac.kr (M.H.J.) 

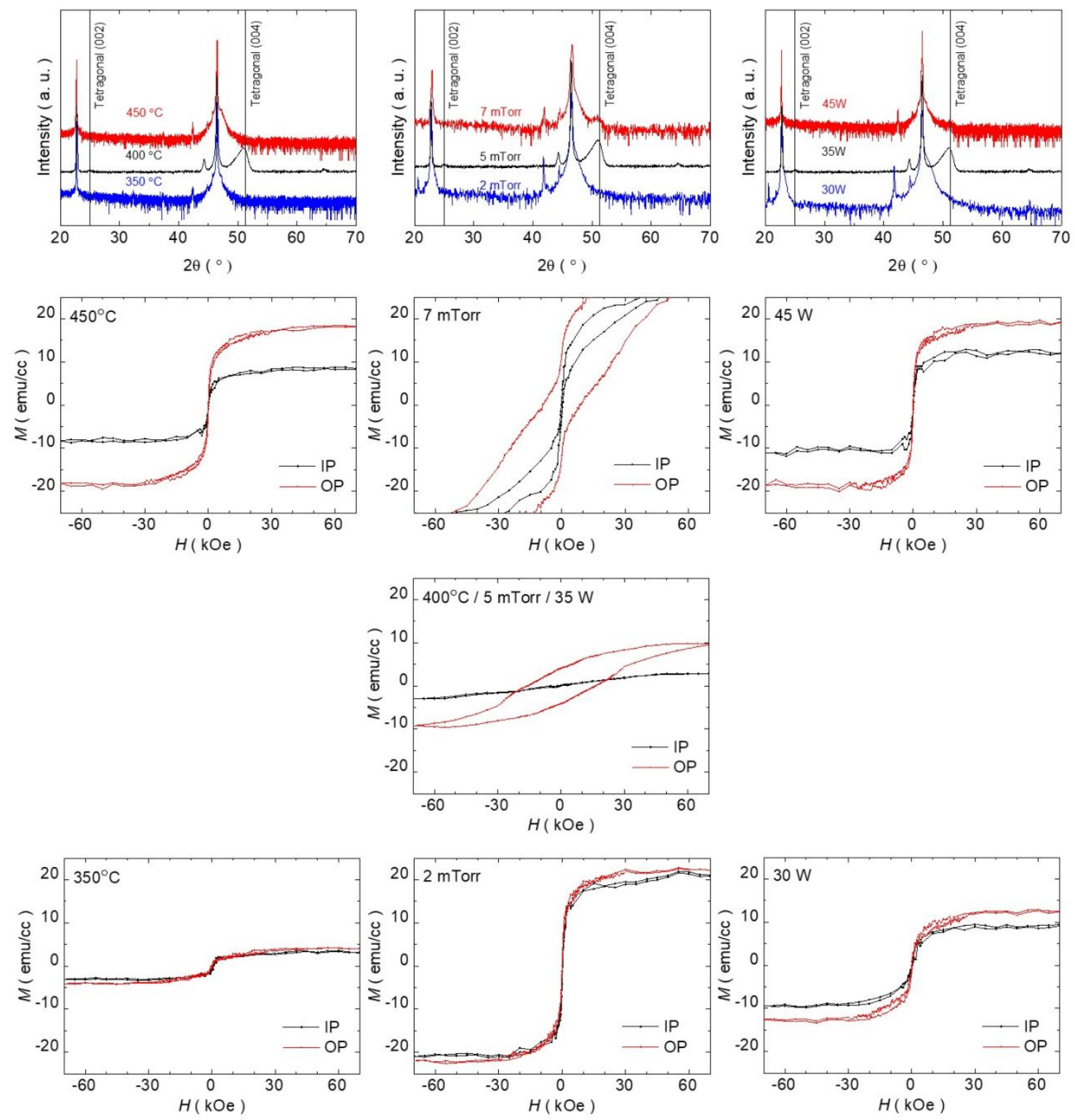

Figure S1. X-ray diffraction patterns and the corresponding magnetic properties of $\mathrm{Mn}_{3-\delta} \mathrm{Ga}$ deposited on STO substrate taken with different conditions of substrate temperature $\left(350-450^{\circ} \mathrm{C}\right)$, argon pressure (2-7 mTorr), and rf gun power (30-45 W). The magnetic field is applied in the plane (IP) and out of plane (OP). The optimal deposition condition for STO is $400^{\circ} \mathrm{C}, 5 \mathrm{mTorr}$, and $35 \mathrm{~W}$. 

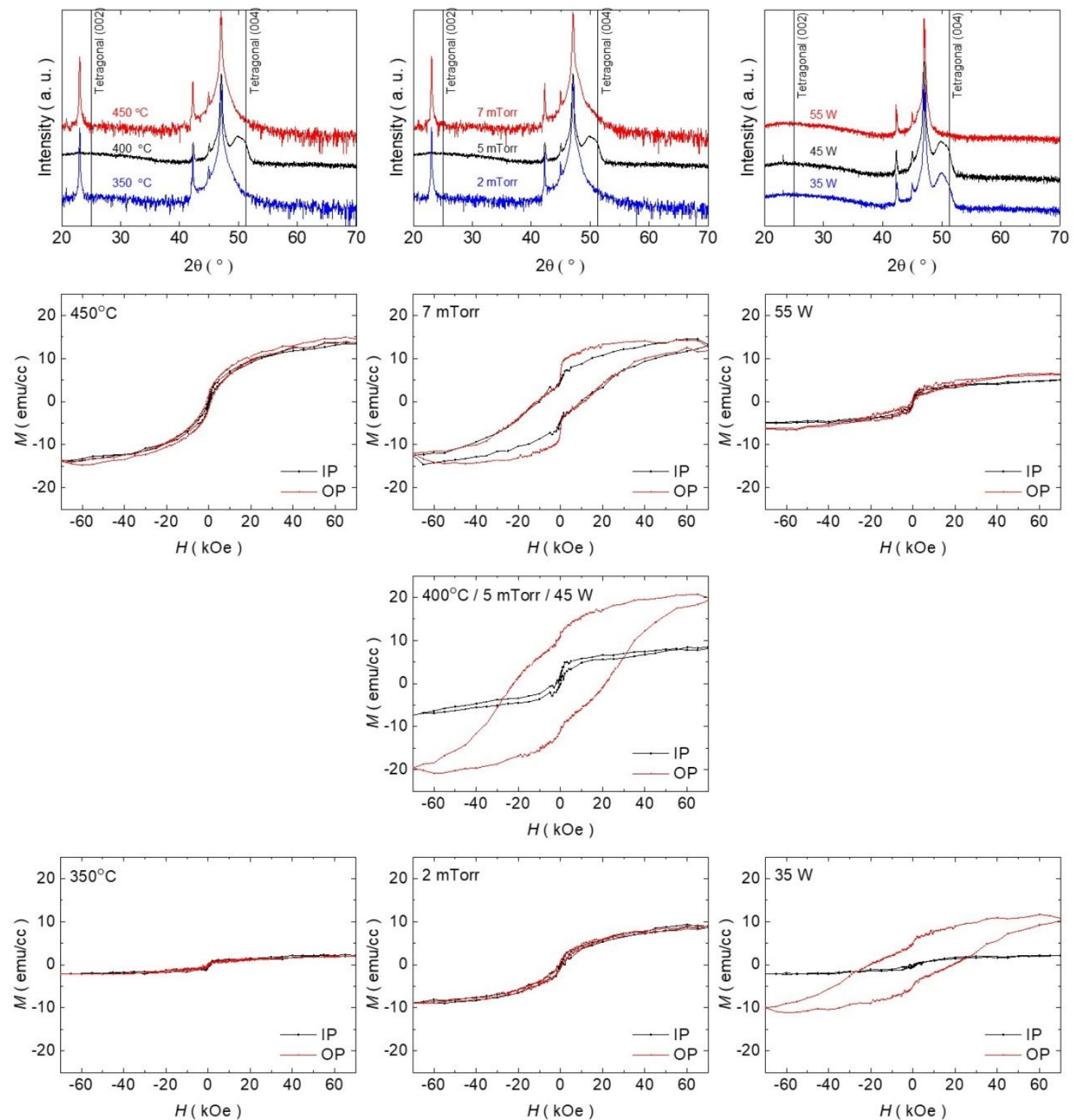

Figure S2. X-ray diffraction patterns and the corresponding magnetic properties of $\mathrm{Mn}_{3-\delta} \mathrm{Ga}$ deposited on LSAT substrate taken with different conditions of substrate temperature $\left(350-450^{\circ} \mathrm{C}\right)$, argon pressure (2-7 mTorr), and $\mathrm{rf}$ gun power (35-55 W). The magnetic field is applied in the plane (IP) and out of plane (OP). The optimal deposition condition for LSAT substrate is $400^{\circ} \mathrm{C}, 5 \mathrm{mT}$ orr, and $45 \mathrm{~W}$. 

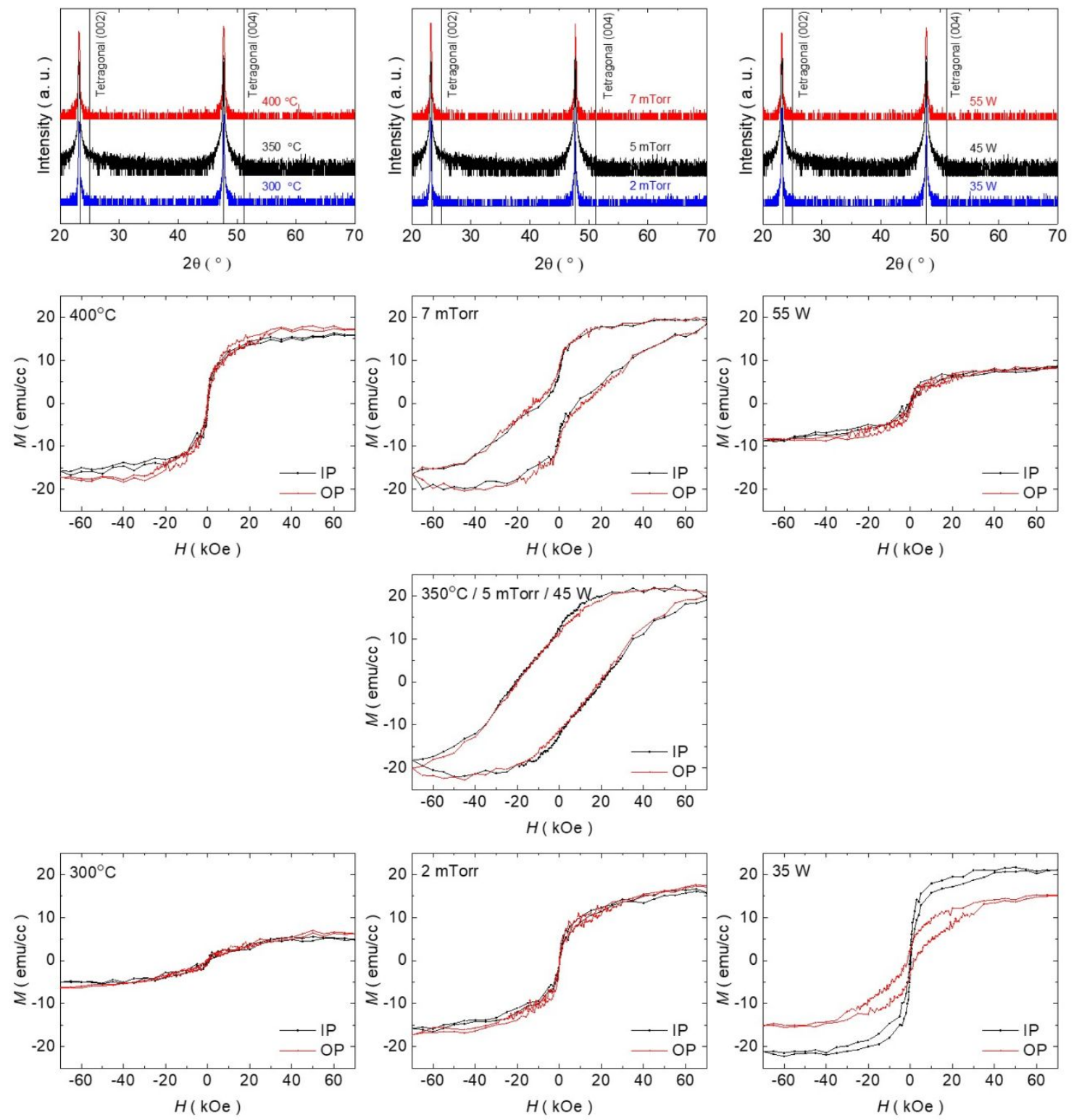

Figure S3. X-ray diffraction patterns and the corresponding magnetic properties of $\mathrm{Mn}_{3-\delta} \mathrm{Ga}$ deposited on LAO substrate taken with different conditions of substrate temperature $\left(300-400^{\circ} \mathrm{C}\right)$, argon pressure (2-7 mTorr), and $\mathrm{rf}$ gun power (35-55 W). The magnetic field is applied in the plane (IP) and out of plane (OP). The optimal deposition condition for LAO substrate is $350^{\circ} \mathrm{C}, 5 \mathrm{mT}$ orr, and $45 \mathrm{~W}$. 